\title{
Didáctica universitaria: ¿un asunto de interés para la universidad actual?
}

\author{
Elisa Lucarelli*
}

\section{Resumen}

Este artículo tiene como objetivo presentar la situación histórica y actual del campo de la Didáctica Universitaria en la Argentina, con especial referencia a los últimos 25 años y en relación con los acontecimientos que se sucedieron en el contexto institucional y nacional. Ejemplifica sus problemáticas a partir de las características y devenir de un programa de investigación en la materia, sus líneas de trabajo y su producción, haciendo énfasis en un estudio que encara en la actualidad acerca de la formación en los aprendizajes complejos de la práctica profesional en el área de la salud, especialmente aquellos que se desarrollan en las Clínicas. Con este propósito utiliza una metodología cualitativa con estudio de casos e instancias de participación. Sus principales resultados dan cuenta de las características que asumen las modalidades de enseñanza en esos ámbitos en lo relativo encarar la tensión que se genera entre la lógica de formación y la lógica de atención de la salud, destacándose la identificación de las modalidades desarrolladas por el profesor, en especial la observación y la supervisión de la tarea de los estudiantes, como práctica real anticipatoria del desempeño profesional. De igual manera fueron reconocidas las modalidades que se generan en relación al lugar del estudiante en la situación de formación y las derivaciones didácticas de la organización en las clínicas y el aprendizaje cooperativo entre estudiantes. Palabras clave: Didáctica - Educación Superior. Intervención Educativa. Innovación en Educación.

* Doctora en Educación pela Universidad de Buenos Aires. Profesora titular regular da Universidad de Buenos Aires. Investigadora y Directora del Programa Estudios sobre el aula universitaria. 
[...] lo que está en juego en esta nueva orientación de las universidades es también, y quizá sobre todo, la capacidad para una sociedad democrática, a pesar de la dinámica de la especialización y a través de la construcción propiamente universitaria de un mundo común de referencias compartidas, de disponer de la posibilidad de un verdadero espacio público. (RENAUT, 2008, p. 70).

\section{La universidad en su contexto}

La universidad en esta primera década del siglo XXI se encuentra inserta en nuevos escenarios signados por múltiples crisis que requieren volver a repensar la institución a la luz de esos cambios de contexto. La debacle originada por el colapso mundial de las economías que afectó el centro del capitalismo, Estados Unidos al comienzo, algunos países de la Unión Europea en la actualidad, parece anunciar el agotamiento del modelo neoliberal conservador, y por ende la regulación de la vida a través del mercado. Más allá de las definiciones de tipo técnico se avizora la necesidad de un debate centrado en las ideas que incluya las distintas dimensiones del mundo social.

La Universidad, acosada por requerimientos de ese entorno para modificar sus prácticas según modelos ajenos a su propia idiosincrasia, parece responder lentamente a la urgencia de plantearse nuevas definiciones que la alejen de pensarse como una institución al servicio de esos modelos.

En América Latina la necesidad de una universidad con pensamiento autónomo y compromiso ético, que se expresa a través de prácticas pertinentes ${ }^{1}$, alejadas de la torre de marfil, se da en tiempos de consolidación de democracias en contexto de pobreza, marcadas por el respeto a la diversidad y con eje en la tolerancia.

Como institución de servicio público y como parte de su compromiso ético la institución concreta su ideario en la resolución de las funciones que le han sido asignadas por la sociedad: junto a la investigación y a la extensión, la formación de los ciudadanos para el desarrollo de profesionales responsables en la dimensión social, con respeto a la dignidad de las personas y los derechos humanos, la democracia, la justicia, la solidaridad. 
Dentro de las ciencias sociales, la Pedagogía y la Didácticas universitarias surgen y se afianzan poco a poco como parte de un espacio de reflexión y de acción para dar respuesta a los problemas que la docencia encuentra cotidianamente en el contexto universitario.

Este artículo se propone abordar el desarrollo de ese campo científico en la Argentina, en el contexto histórico y actual de las universidades públicas, considerando cómo la preocupación por las innovaciones en la enseñanza universitaria está presente en el ideario de la Reforma Universitaria de 1918 y en la producción pedagógica de mediados de ese siglo. Se plantea también analizar a la Pedagogía y a la Didáctica Universitarias como espacios disciplinares profesionales que comparten perspectivas y problemáticas comunes, no obstante la diferenciación de sus objetos de estudio y a la vez considerar las características de investigaciones centradas en las prácticas del aula universitaria

\section{La universidad argentina frente a los problemas pedagógicos}

El campo de la Pedagogía y la Didáctica Universitarias argentinas se desarrolla en circunstancias de peculiares modificaciones en la estructura general del nivel superior y en especial en su normativa, a partir del reconocimiento de las repercusiones que han tenido sobre la identidad institucional las reformas realizadas en los 90 y del debate actual acerca de una nueva Ley de Educación superior que supere esa situación.

En efecto, la educación superior en la Argentina se ha visto impactada en los últimos ańos del siglo XX por reformas, que reinterpretaron en el contexto local, políticas de índole mundial, orientadas por agencias de financiamiento siguiendo premisas tales como el desplazamiento del Estado como garante del servicio y de su financiamiento y el protagonismo del mercado en la generación y satisfacción de nuevos consumidores en el nivel superior (MOLLIS, 2008, p. 26).

Algunos datos históricos permiten situar esta problemática. La rica trayectoria de las universidades argentinas, se inicia en el siglo XVII con la creación de la Universidad de Córdoba, en la época colonial, se continúa en el XIX, ya lograda la independencia política de España, con la creación de la Universidad de Buenos Aires en 1821. Estos centros, 
junto a otros establecidos al comienzo del siglo XX, estaban en manos de grupos de elite, concentrando el ingreso a las aulas y el gobierno universitario. Es el movimiento de la Reforma Universitaria, originado en 1918 en la Universidad de Córdoba, el que, conjugando requerimientos políticos, administrativos y pedagógicos, provoca la finalización del dominio del poder por parte de sectores oligárquicos y conservadores y la inclusión en el ámbito y gobierno universitarios, de sectores medios en proceso de ascenso social y político en un contexto favorable de ampliación política en el país. Los principios reformistas, expandidos casi en simultaneidad en varios países de América Latina, ${ }^{2}$ se refieren a aspectos políticos y sociales trascendentes para la vida de la institución, como son la autonomía universitaria, el cogobierno de los estudiantes junto a los docentes y a otros sectores universitarios, la coexistencia de la universidad profesionalista con la científica, la revalorización de la función social de la institución, la extensión, la solidaridad con el pueblo, el compromiso con el cambio social (FERNÁNDEZ LAMARRA, 2003, p. 30); pero también introduce cuestionamientos y reclamos que hacen a la faz pedagógica de la vida universitaria, abriendo las puertas a los cambios en las aulas: la existencia de las cátedras libres, la renovación pedagógica, la centralidad académica de los estudiantes, la creación de una carrera académica del profesorado, los concursos para acceder al cargo docente. A la vez se instala como principio de excelencia sostenido por el movimiento reformista transformar a la universidad en un centro de investigación científica, sosteniéndose la necesidad de que los profesores, a la par, que enseñar debían investigar. ${ }^{3}$

Se instala de esta manera en las instituciones la representación del docente investigador que imprime los mecanismos de selección y ascenso del profesorado. Esta situación se mantiene hasta nuestros días intensificada por disposiciones normativas y reguladoras tales como los programas de estímulo a la docencia, que privilegian los antecedentes producidos por la investigación por sobre aquellos que aluden a la capacidad pedagógica de los docentes, marcando un terreno de contradicciones en la conformación del rol del profesor universitario.

Las consecuencias de estos principios reformistas se transvasaron con distinto ritmo y con diversos énfasis en la cotidianeidad de las aulas en las universidades públicas argentinas en los períodos democráticos de la historia 
argentina del siglo pasado, siendo aquellos negados durante los regímenes militares, en los que queda avasallada la autonomía universitaria.

El debate y la reflexión acerca de los principios pedagógicos se desarrollaron fundamentalmente a través de la acción de filósofos y políticos, quedando en segundo plano la conformación de un corpus sistemático acerca de qué es enseñar en la universidad. No es sino hasta fines de la década del 1950 en que comienza a ser preocupación el desarrollo de recursos genuinamente pedagógicos en la universidad. Es en esta época donde se advierten esfuerzos institucionales organizativos concretados en Unidades de Pedagogía Universitaria y de Orientación Vocacional que brindaban apoyo a estudiantes y profesores universitarios especialmente en las Universidades Nacionales del Litoral (UNL) y Buenos Aires (UBA). Coincide esta época con el surgimiento de un pensamiento latinoamericano en el campo de la ciencia y la tecnología y de la búsqueda por parte de la universidad en transformarse en actor de la planificación del desarrollo nacional (LANGER, 2008).

La universidad científica, de tradición humboldtiana, se vuelve hacer presente manifiesta en la Argentina oponiéndose al modelo exclusivamente profesionalista; se valora el profesor como investigador y se apoya el desarrollo de los laboratorios y de los institutos de investigación. Se propicia la modernización de los planes de estudio y la diversificación de las ciencias humanísticas y sociales, dando lugar a la creación de las carreras de Sociología, Psicología, Antropología y a la transformación de la carrera de Pedagogía, de fuerte énfasis filosófico, en la de Ciencias de la Educación, articulada a partir de entonces con el campo social.

En simultáneo las Unidades de Pedagogía Universitaria y de Orientación Vocacional de la década del 1960 generan un significativo avance de la presencia de lo pedagógico y didáctico en la universidad argentina, y se concreta a través del surgimiento de grupos de profesionales provenientes de las ciencias sociales, pedagogos, psicólogos, sociólogos, preocupados por los procesos que se dan en el aula universitaria. Estos grupos (que desarrollan principalmente actividades de formación de los docentes universitarios en las temáticas de enseñanza, aprendizaje, currículum, las relaciones de la educación con el contexto), derivarán más adelante en los equipos de asesoría pedagógica universitaria. 
También, desde la reflexión sistemática, aparecen en la Argentina obras de pensadores que hacen sus aportes a la construcción del campo pedagógico universitario. Ricardo Nassif, Risieri Frondizi, Gregorio Weinberg y Pedro Lafourcade posibilitan a través de sus obras a una mejor comprensión de esos procesos que se articulan en la institución en torno a la formación.

Me detendré en el pensamiento de Nassif (1974), quien planteó, desde una dimensión teórica, el lugar de la Pedagogía Universitaria y su valor para la construcción de una universidad de cara a su transformación. En su texto Pedagogía universitaria y construcción de la universidad, trabaja los diversos enfoques con que se puede abordar el problema de la Universidad. Nassif (1974) clasifica los enfoques en tradicionales (el filosófico y el histórico), actuales (político, sociológico y económico) y potenciales. Dentro de estos, (definidas como aquellos de desarrollo incipiente en esos tiempos), incluye el geográfico, el administrativo, el psicológico y el pedagógico, señalando que sobre éste se construye la Pedagogía universitaria. En ese mismo trabajo avanza sobre la asociación entre innovación y universidad al señalar que:

una estrategia transformadora tiene que partir del reconocimiento de la realidad temporal y cambiante de aquellas instituciones tendiente a encontrar estructuras que les permitan adecuarse creadoramente a los estadios multiformes de la historia humana, en general, y de cada uno de los pueblos en particular. (NASSIF, 1974, p. 401).

Destaca Nassif la centralidad de una renovación pedagógica de la universidad para el cambio en la institución, al expresar:

Esta nueva idea de la Universidad es la que ha impuesto la existencia de una pedagogía universitaria que renueve viejos puntos de vista y amplíe sus dominios hasta tocar los mismos límites de la educación en cualquiera de sus aspectos. Una pedagogía universitaria renovada abarcará tópicos de la más diversa índole cuyo número variará según el criterio que se adopte, pero siempre sobre el supuesto de que la Universidad como organismo educativo encontrará apoyo en fundamentos pedagógicos expresos y científicamente logrados. (NASSIF, 1974, p. 407). 
En su obra póstuma, Teoría de la educación, Nassif (1980) insiste en la necesidad de articular enfoques para la comprensión de la complejidad de los procesos universitarios en su dimensión pedagógica. $\mathrm{Al}$ respecto señala que:

La justificación primera de un enfoque pedagógico y de una Pedagogía de la Universidad no es otro que la naturaleza formativa de ésta. Un hecho simple que, sin embargo, no es todavía - a pesar de los avances realizados - aceptado con la generalidad deseable. De donde, curiosamente, la primera misión de la mirada pedagógica sobre la Universidad ha de ser la de redescubrir la funcionalidad educativa de esta, y evidenciarla como uno de los determinantes de su constitución y de su desarrollo históricos. (NASSIF, 1980, p. 311).

La búsqueda de especificidad de una reflexión sistemática desde la multidimensionalidad sobre los problemas relacionados con la formación universitaria, se refleja también en esta obra del pedagogo argentino, quien expresa:

Aún con limitaciones que toda pedagogía naturalmente tiene, la universitaria logra significado preciso en tanto, dada la pedagogicidad de su campo, hace viable una conjunción de los otros enfoques, sin despojarlos de su personalidad y orientándolos en la dirección de una de las funciones nucleares de la Universidad. Ciertamente que no todos los problemas universitarios son pedagógicos, mas aún aquellos que escapan al quehacer pedagógico pueden ser comprendidos y, sobre todo, instrumentados por este desde su puesto de observación. Si algo es claro en Pedagogía universitaria es que, sean o no específicas las cuestiones de que trata, todas han de entrelazarse en una visión unitaria de la Universidad. Si esta visión falta, la nueva Universidad seguirá siendo un ideal lejano, inalcanzable. (NASSIF, 1980, p. 311-312).

Al mismo tiempo que Nassif se refería explícitamente a la necesidad de un campo de reflexión sistemática de Pedagogía Universitaria, Pedro 
Lafourcade, en la Universidad Nacional de San Luis, desarrolla un espacio de investigación y de práctica en torno a los problemas de la enseńanza en la universidad, y escribe un texto referido específicamente a estos asuntos, que se constituye en una obra de referencia (LAFOURCADE, 1974) para la constitución del campo de la Didáctica Universitaria. Temáticas en torno al aprendizaje, el currículum, su planeamiento a nivel de asignaturas, las estrategias docentes de enseñanza y de evaluación son trabajadas por el autor desde la perspectiva de la institución universitaria y su diversidad disciplinar.

En la Argentina los desarrollos generados acerca de la formación y la enseńanza en la universidad, desde una perspectiva dinámica y contextualizada, se interrumpieron en los períodos de dictadura, durante los cuales se manifiestan otros encuadres, propios del pensamiento único; según ellos lo pedagógico se reduce a la búsqueda de procedimientos tecnicistas sobre cómo operar en el aula.

En la década del 1970, en la región latinoamericana, es México el país donde se fortalece el campo de la Pedagogía y la Didáctica Universitarias y adonde acuden muchos de los intelectuales de países, como la Argentina, que viven regímenes dictatoriales. La producción mexicana acerca de la formación de docentes en las diversas áreas disciplinares profesionales, el desarrollo de formas curriculares que se articulan en consideración de las demandas sociales, la investigación sobre perspectivas teóricas críticas en los programas de estudio, las reflexiones sobre mecanismos de poder, enseńanza y currículum, han conformado un pensamiento pedagógico propio en torno a la universidad. Las repercusiones de los dramáticos sucesos de Tlatelolco derivaron en transformaciones importantes en las grandes universidades del país (Universidad Nacional Autónoma de México, Universidad de Guadalajara), con la desconcentración en nuevas Unidades (Escuelas Nacionales de Estudios Profesionales) y especialmente en cuanto a la creación de institutos dedicados al desarrollo de programas masivos orientados a la preparación pedagógica de los docentes, a las nuevas tecnologías y la reforma de los planes de estudio, así como a la producción de conocimiento acerca de la universidad. ${ }^{4}$ Se crean asimismo nuevas universidades con estructuras político administrativas innovadoras y descentralizadas (tal es el caso de la Universidad Autónoma Metropolitana), donde la tríada docenciainvestigación-servicio se articula con las políticas sectoriales locales, dando lugar, consecuentemente al diseño de propuestas curriculares alternativas. 
Las obras de Díaz Barriga Didáctica y currículum (1984) y Ensayos sobre la problemática curricular (1990), revelan parte de ese pensamiento, en el que se incluye también la producción de Furlán, Remedi, de Alba, entre otros. Es precisamente este grupo, liderado por Alfredo Furlán el que elabora una obra (creemos que la primera de este período), "Aportaciones a la Didáctica de la Educación superior" dedicada a "contribuir a la discusión de algunos nudos conceptuales y técnicos de la didáctica de la educación superior" (FURLÁN, 1979, p. 5) y en la que se sostiene una perspectiva contextualizada del aula universitaria, en un encuadre cercano a la ciencia social crítica y a la psicología profunda, perspectiva esa opuesta al encuadre tecnicista en vigencia en muchas de las instituciones de la época.

En Argentina, a partir de 1984, con la recuperación de la democracia y de la autonomía universitaria, el interés por la formación y la enseñanza en la universidad se intensifica, concretándose en el desarrollo de un campo de producción científica, como es el de la Pedagogía y la Didáctica Universitarias, alrededor del tema, y de prácticas orientadas a la innovación en las prácticas institucionales.

\section{Perspectivas comunes y objetos diferenciados}

Vale considerar cómo entendemos la Pedagogía y la Didáctica Universitarias hoy.

En primer lugar cabe destacar, sin entrar en preciosismos semánticos, pero sí intentando evitar confusiones epistemológicas, que si bien ambas disciplinas se refieren al estudio sistemático de los problemas relacionados con la función docente de la universidad, existen entre ellas matices distintivos provenientes de la especificidad de las Ciencias de la Educación y que se relacionan con sus respectivos objetos de estudios, la formación y la enseñanza. Así afirmamos que la Pedagogía Universitaria se define como un espacio de conocimiento orientado a la comprensión de los procesos de formación que se dan en la universidad, a partir de la consideración de los sujetos involucrados, su relación con el contexto y con los otros procesos que se desarrollan en ese ámbito, mientras que la Didáctica Universitaria analiza el proceso de enseñanza que un docente o un equipo de docentes organiza en relación con los aprendizajes de los estudiantes, en función de un contenido científico, tecnológico o artístico - altamente especializado, - 
orientado hacia la formación en una profesión (LUCARELLI, 1998, 2007, 2009). Desde una perspectiva multidimensionada, histórica y situacional, incluye, como estructurantes junto al contenido disciplinar y profesional, al estudiante y sus aprendizajes enmarcados en el contexto institucional y social del que forma parte.

Es así que cabe reconocer que ambas disciplinas se interesan, desde distintos ángulos, por los procesos que afectan la función docente de una institución que caracterizada, desde su creación, y tal como se recordaba anteriormente, como un centro de formación centrado en la producción de conocimientos

Es necesario aceptar que si bien la presencia de la dimensión pedagógica y didáctica es explícitamente demandada y lentamente aceptada por la institución, la identificación de los saberes que un docente debe dominar para enseñar en este ámbito sigue hegemonizada por los contenidos disciplinares profesionales sustantivos; esta situación da cuenta a la vez una posición epistemológica en la que el contenido producido por las ciencias duras es el de mayor reconocimiento, mientras que lo pedagógico, como proveniente del campo de las ciencias sociales es escasamente valorado. ${ }^{5}$ Los contenidos sustantivos son los propios de la comunidad profesional de origen del docente, de la tribu académica en la que se formó y a la cual reconoce como propia. De allí proviene su poder y prestigio, no de la docencia universitaria como saber pedagógico (LUCARELLI, 2006). La práctica profesional con la que se identifica el docente universitario se tensiona, además, en un terreno definido por la multiplicidad de prácticas, la práctica profesional para la cual fue formado, la práctica de enseñar en la que es seguramente idóneo pero que debe ejercer cotidianamente, la práctica de investigar en su propio campo disciplinar profesional, con mayor reconocimiento en términos de ingreso y ascenso en la docencia

En este contexto, difícil y contradictorio, la Pedagogía y la Didáctica Universitaria va configurando su identidad, sin que la diferenciación de los dos ámbitos disciplinares sea percibida aún dentro del campo de las Ciencias de la Educación, considerándoselos muchas veces como equivalentes para referirse al abordaje sistemático de los problemas educativos en general de la universidad. ${ }^{6}$

Dentro del ámbito de las carreras de Ciencias de la Educación, como parte de las tensiones propias de un campo profesional nuevo, con fronteras 
externas lábiles, y con una estructuración interna de escasa determinación, se percibe la permanencia de una representación social del área didáctica como un enclave tecnicista, ajeno a las definiciones del contexto. Esta mirada anacrónica sobre lo didáctico, así como sobre otras disciplinas referidas a la subjetividad, ${ }^{7}$ proviene de sectores académicos, que pertenecientes a las disciplinas atingentes a lo macroeducativo, tales como sociología, historia, política educativas, pretenden ser hegemónicos en el campo, y reducir la importancia de espacios curriculares responsables de analizar e intervenir en las prácticas de enseñar y aprender. Esta pretensión desconoce las nuevas perspectivas que sobre estas prácticas desarrolla la Didáctica fundamentada crítica, definida por la multidimensionalidad, la contextualización, la consideración de los fundamentos, la búsqueda de estrategias apropiadas y el interés por la articulación teoría-práctica (CANDAU, 1995). La Didáctica Universitaria es también depositaria de esa mirada prejuiciosa sobre su campo de producción de conocimiento y de intervención, homologándolo a un mero campo instrumental. ${ }^{8}$

En un grado de significación menor pero que hace también a la escasa visibilidad del campo dentro del ámbito universitario son frecuentes las imprecisiones, en el lenguaje habitual docente, en cuanto a la confusión entre disciplina y objeto de estudio. Así hay referencias a la Pedagogía y/o a la Didáctica universitarias entendiéndolas con el ejercicio profesional de un profesor en la universidad, "la pedagogía universitaria es llevada a cabo por docentes que no tienen identidad única”, "en las clases de estos profesores se evidencia una inadecuada didáctica”.

Consideramos que sólo a través de un mayor desarrollo de producción científica y de intervención en los procesos educativos de la vida institucional, es que el campo pedagógico y didáctico se puede ir consolidando y logrando identidad en la institución universitaria.

\section{Desarrollos actuales de la Didáctica Universitaria en Argentina}

En la Argentina el campo de la Didáctica Universitaria se desarrolló desde el inicio del actual período democrático en 1984 a través de tres vertientes: las actividades de formación, intervención en el aula e investigación desarrolladas por las asesorías pedagógicas en las universidades 
nacionales, la instalación de los Programas de Maestría en Docencia Universitaria, Didáctica y afines, y la producción llevada a cabo por los equipos de investigación dedicados particularmente a las problemáticas de la enseñanza en la Universidad.

La asesoría pedagógica universitaria es reconocida como una profesión de ayuda a las distintas instancias institucionales, desde los docentes a la gestión, a través de prácticas de formación, intervención e investigación orientadas a favorecer cambios que afecten a la institución educativa como un todo y al aula en particular.

En este sentido el asesor pedagógico universitario se manifiesta como un animador de las innovaciones, en la función de acompañamiento al desarrollo de las prácticas de otros, y a la vez como sujeto protagónico en la construcción del campo de la Didáctica Universitaria, a través de la reflexión sobre esas prácticas y el desarrollo de indagaciones sistemáticas sobre la enseñanza.

A su vez estas prácticas son reveladoras de una determinada concepción pedagógica y didáctica, desde donde se definen rumbos y decisiones. Su trabajo cotidiano da cuenta de la existencia de ese marco teórico valorativo, que al mismo tiempo que ordena, clarifica y fundamenta las acciones, permite definir qué decisiones se toman y por qué se eligió ese camino de acción y no otro para intervenir en los procesos que se dan en el aula.

En un trabajo sobre la actividad de asesoramiento en las instituciones educativas, Lidia Fernández (1982, p. 63), sostiene que el pedagogo en esos establecimientos puede contribuir a un aumento de la eficiencia institucional a través de un mejoramiento de aspectos que hacen a la organización y formación docente, a la vez que mediante su participación en la atención a un conjunto de variables relativo al campo didáctico pedagógico, tales como: la especificación de fines y su traducción en un modelo didáctico, el análisis de las condiciones de los alumnos para desarrollar el proceso de aprendizaje, la programación de un curriculum que permita alcanzar esos objetivos con esos alumnos, la creación de sistemas de evaluación que permitan la supervisión y el control, la preparación de docentes y directivos para asumir sus roles y la ayuda a los estudiantes, a través de los docentes, para ajustar los suyos propios. 
En el caso específico del asesor pedagógico en las universidades, el rol adquiere los rasgos que definen el campo académico, (y por tanto se ve afectado por el juego de tensiones y luchas que determinan su estructuración), ya que debe operar en una comunidad disciplinar profesional que no es la propia. Del reconocimiento de esta situación, se derivan principios que deberían caracterizar su tarea en las distintas unidades académicas (GARRAMUÑO, 2000). Uno de esos principios es el de aceptación de la diversidad, (esto es la posibilidad de entender al hombre como sujeto inmerso en un mundo también diverso); este reconocimiento le permitiría al asesor pedagógico, estar atento a las necesidades de uno y de los otros, aportar desde las demandas, trabajar desde espacios no formales, elementos estos que sostienen el trabajo para la diversidad.

El otro concepto clave para trabajar con comunidades disciplinares profesionales en la universidad es el de extranjeridad, esto es la necesidad de tomar conciencia de la condición de extranjero que tiene el asesor pedagógico universitario en una unidad académica que no es la de su grupo profesional de procedencia, sino de lo médicos, los economistas, los físicos... Esta es una condición favorable ya que aminora la noción de extrañamiento que puede generar su inserción; el ser conciente de esa condición, el aceptarse como extranjero le posibilita instalarse y ejercer la intervención o las actividades de formación docente desde otro "lugar" con menores dificultades."

En las universidades argentinas en la actualidad las prácticas del asesor pedagógico se centralizan en acciones ligadas a la formación de los docentes, de intervención en la vida de las aulas, de investigación, y también algunas de apoyo administrativo y de rutina burocrática; otro rubro importante lo constituye el apoyo a la gestión, hoy hegemonizada por los programas de acreditación de carreras y de evaluación institucional. ${ }^{10}$

En Argentina el desarrollo de Programas de Especialización y Maestrías en Ciencias Sociales, y en Educación en particular, es reciente, en relación con otras universidades del continente, ya que se inició hace alrededor de treinta años. A partir de entonces el campo de posgrados se ha diversificado dando lugar a la instalación de programas en Docencia Universitaria, Didáctica y afines; estos programas que integran dentro de sus participantes a docentes de distintas profesiones y especialidades, son de por sí, en sus prácticas cotidianas de formación, un interesante laboratorio de producción de 
Pedagogía y Didáctica Universitarias, acrecentado por las investigaciones que sirven de eje a la elaboración de las tesis respectivas.

En paralelo con esto, en casi todas las universidades del país se han ido conformando equipos de investigación interesados por las problemáticas de la educación universitaria en general y particularmente, de las prácticas en el aula. Estos equipos son integrados en algunos casos por especialistas en Didáctica provenientes del campo de las Ciencias de la Educación, pero en gran proporción se trata de grupos de investigadores disciplinares, que solos o incorporando un didacta en algunos casos, desarrollan estudios sistemáticos sobre la enseñanza en los diversos campos disciplinares, dando lugar así al incremento de la producción de las Didácticas Específicas universitarias. Los programas de incentivos a la investigación, como estímulo económico a esta producción y de categorización de los académicos han contribuido también, aunque de manera vicaria, a este desarrollo de la investigación educativa.

\section{Un programa de investigaciones centrado en las problemáticas de la Didáctica Universitaria}

Parece importante ilustrar este camino en la conformación del campo de la Didáctica Universitaria a través de presentación del recorrido realizado por un Programa de investigación en el área de Didáctica Universitaria en sus 25 ańos de existencia en una universidad pública argentina.

El Programa "Estudios sobre el aula universitaria" del Instituto de Investigaciones en Ciencias de la Educación de la Universidad de Buenos Aires se creo al ańo siguiente de la recuperación de la democracia argentina en consonancia con las políticas ese Instituto. En efecto en la Universidad de Buenos Aires el proceso de restauración de las condiciones académicas y de gobierno de la institución, con la puesta en vigencia del Estatuto Universitario, se da paralelamente a las acciones tendientes a la normalización del claustro docente y al inicio de programas orientados a la búsqueda de fortalecimiento de la investigación y de una mejor calidad de la enseńanza. Dos de las acciones tendientes a este último propósito fueron el Programa de Reforma Curricular, impulsado por el Rectorado, y la creación de las Comisiones Asesoras Pedagógicas en las distintas Facultades.

Animado por este espíritu democrático, el entonces nominado Instituto de Ciencias de la Educación ${ }^{11}$ reinicia sus acciones y propone la apertura 
de la sede a la comunidad educativa en su conjunto. Consecuentemente, convoca para la elaboración de su Plan de trabajo correspondiente a 1985 "a quienes se hallan directa o indirectamente involucrados en el quehacer educativo" (SIRVENT, 1985, p. 1). Considera esta una forma concreta de ir desarrollando los lineamientos de política de investigación propuestos: "Desarrollar un trabajo de alto nivel científico a la vez que comprometido con la realidad social, política y económica de nuestro país [...] [como] aporte en el camino de disminuir el hiato existente entre el trabajo científico y la práctica educativa” (SIRVENT, 1985, p. 1-2).

Como una expresión de ese compromiso con su contexto inmediato, el Instituto comienza a desarrollarse un programa de apoyo a la Reforma Curricular de la Universidad, centrado en el desarrollo de encuentros entre docentes y otros actores institucionales de las distintas unidades académicas (en especial asesores pedagógicos e investigadores del Instituto), donde se presentan y analizan factores facilitadores y escollos para la concreción de dichas modificaciones. Se abordan diversas temáticas relacionadas con el aprender y enseñar en la universidad, en situación de numerosidad en las aulas, tales como los problemas relacionados con sistemas de evaluación, utilización de estrategias didácticas apropiadas, inclusión de las nuevas tecnologías, articulación con la escuela media, adecuación del contenido científico, entre otros. ${ }^{12}$

Muy pronto los docentes que asisten a "Talleres de reflexión de apoyo a la reforma curricular de la UBA" (años 1985 y 1986) van centrando sus discusiones en el análisis de las prácticas de enseñanza; el relato de sus estrategias para afrontar, de manera alternativa a la habitual, los problemas que se le presentan en el aula constituye la temática privilegiada de esas reuniones. La cuestión de los procesos alternativos de gestación de prácticas innovadoras protagónicas, simultáneamente a la implementación de reformas curriculares impulsadas por la administración, surge como problema de interés para posibles investigaciones y para el desarrollo de acciones participativas de intervención en las prácticas docentes. Es así que un equipo de investigadores del ICE y asesores pedagógicos de las diversas Unidades Académicas de la UBA toman este tema como eje para la investigación y el desarrollo de acciones de formación en el campo de la Didáctica Universitaria. Este segundo momento culmina con la organización de un Proyecto específico de investigación que, considerando las prácticas 
innovadoras y su difusión como problema central, se integran al Proyecto Institucional del ICE de "Democratización de la Educación, obstáculos y alternativas". Este se propuso abordar, desde distintas dimensiones, las características que asumen los espacios públicos de educación en los primeros años del retorno a la vida democrática del país y de la universidad. Este Proyecto específico, centrado en la vida de las cátedras, acompaña el devenir de una institución, la UBA, preocupada, desde el retorno de la vida democrática, por el mejoramiento de los procesos pedagógicos a través de diversas estrategias; algunas de ellas fueron: el Programa de capacitación docente que acompañó los primeros años de implementación del Ciclo Básico Común, la creación de las Comisiones Asesoras Pedagógicas como apoyo al desarrollo de las prácticas del enseñar y el aprender en el contexto de cátedras y departamentos, de las Facultades, y el abordaje, desde la investigación, de aspectos relacionados con la masificación y la calidad de la enseñanza.

A partir de la década del 90 el Programa, en un tercer momento, encara tres líneas diferenciadas y a la vez articuladas de acción: la realización de Jornadas de difusión y análisis de experiencias innovadoras en conjunto con el Proyecto de Pedagogía Universitaria, ${ }^{13}$ de Jornadas de docentes (Expocátedras), la formación de asesores pedagógicos universitarios, y, paralelamente a ellas, el desarrollo de investigaciones sobre ambas temáticas: la innovación en el aula universitaria centrado en la relación teoría-práctica y las características que definen el rol del asesor pedagógico en la universidad..

En las investigaciones acerca de la innovación en las aulas universitarias reconocemos como innovación a la práctica de enseñanza o de programación de la enseńanza, en la que, a partir de la búsqueda de la solución de un problema detectado, se produce una ruptura en las prácticas habituales que se dan en el aula de clase, afectando el conjunto de relaciones de la situación didáctica (LUCARELLI, 2009).

Aparecen entonces en este concepto ciertos rasgos que queremos destacar: ruptura, protagonismo, carácter sinérgico de la articulación de teoría y práctica en las innovaciones.

La innovación como ruptura, implica la interrupción de una determinada forma de comportamiento que se repite en el tiempo, de una praxis repetitiva en palabras de Agnes Heller (1977) esto es aquella que reitera esquemas prácticos desarrollados precedentemente y que incluye 
el pensar repetitivo, un proceso mental sumamente abreviado, y que se corresponde casi con un sistema de reflejos condicionados. La innovación como praxis inventiva se contrapone con esta forma de pensamiento, apelando al pensamiento creativo y divergente.

Toda innovación forma parte de una trama experiencial, por tanto se legitima, dialécticamente, con la posibilidad de relacionar esta nueva práctica con las que ya dispone el sujeto, a través de mecanismos de oposición, diferenciación o articulación. Una práctica innovadora de la enseñanza puede ser entendida solamente en el contexto de la historia de los sujetos, los grupos o las instituciones que la portan, esto es, si se la analiza como parte del conjunto de prácticas que desarrolla un determinado actor; se parte del supuesto que ese comportamiento cobra sentido a la luz del repertorio de formas de actuar que ha desarrollado ese sujeto a lo largo de su vida (BLEGER, 1971), en este caso en lo referente al desempeńo de su rol docente. Es en ese sentido que sostenemos que las innovaciones son protagónicas es decir, el primer actor, el protagonista es el sujeto social que sostiene esa práctica desde su gestación en la percepción del problema, y no un mero ejecutor de soluciones pensadas por otros. Por otro lado si bien una experiencia innovadora se gesta alrededor de la solución a un problema puntual, específico centrado, por ejemplo, en la organización del contenido o en la necesidad de introducir nuevas tecnologías de la comunicación, o de modificar las estrategias de enseńanza, para ser innovadora tiene que afectar al conjunto de las relaciones que se dan en la situación didáctica curricular, dando lugar a un proceso en el que se articulan, dialécticamente, momentos teóricos y momentos prácticos. En el proceso de ruptura con los modos habituales de operar que originan innovaciones en el aula universitaria, la articulación teoría-práctica funciona como un elemento sinérgico de la situación didáctica.

Las investigaciones alrededor de esta temática de la innovación dieron lugar al trabajo conjunto, desde 1992, con un grupo de investigadores de Universidades de la Región Sur de Brasil, dirigidos por María Isabel da Cunha (UFPel/Unisinos) y Denise Leite (UFRGS). En la actualidad el Programa continúa desarrollando acuerdos y actividades de intercambio con la Rede Sulbrasileira de Pesquisadores da Educaçao Superior, (RIES) que en los últimos ańos ha llevado a cabo importantes investigaciones en el campo de la Pedagogía Universitaria. 
En un cuarto momento que se puede ubicar a partir del año 2000, el Programa, interesado por las prácticas innovadoras de los docentes universitarios, profundiza su preocupación por indagar las modalidades de enseñanza que se desarrollan alrededor de la formación de aprendizajes complejos propios de la práctica profesional en la formación de grado de la Universidad de Buenos Aires, en especial en las áreas relacionadas con las Ciencias Veterinarias, la Odontología y la Medicina. ${ }^{14}$

En la actualidad se está trabajando en el estudio de los modos en que se manifiesta la articulación teoría-práctica, preguntándose acerca de las modalidades que desarrollan los docentes universitarios a través de la puesta en acción de dispositivos metodológicos de enseñanza y evaluación que se dan particularmente en las asignaturas del Ciclo superior de las carreras de formación de odontólogos y médicos que son desarrolladas en el espacio particular de las Clínicas, Consultorios y Salas de internación de las Unidades Docentes Hospitalarias.

Estos ámbitos, como forma particular del aula universitaria abren la puerta al estudio de nuevas cuestiones ausentes de la agenda tradicional de la Didáctica. Considerados desde un ángulo no habitual permiten estudiar la tensión que se genera entre la lógica de formación y la lógica de atención de la salud en la tarea de enseñanza, las modalidades centradas en la actividad que desarrolla el profesor, en especial la observación y la supervisión de la tarea que realiza el estudiante, como práctica real anticipatoria del desempeño profesional; las modalidades que se generan en relación al lugar del estudiante en la situación de formación y las derivaciones didácticas de la organización en las clínicas y el aprendizaje cooperativo entre estudiantes.

Así considerado, esta nueva configuración del espacio que supone la formación en Clínicas templa de un modo peculiar la relación entre profesión, institución y enseñanza. La identificación de la importancia que adquieren los componentes emocionales y valorativos en esos ámbitos, en relación con aspectos de significado profundo son como el respeto al paciente, el compromiso con la vida, el lugar de la prevención, el significado de la contención; la constatación de que esos contenidos no son considerados como tales en la programación curricular y en su puesta en acción; los desafíos y exigencias (de lo que no siempre se hace conciencia) que significa asumir el rol docente para el personal de salud no inserto de manera regular en la institución formadora, son algunas de 
las problemáticas que atraviesan la investigación didáctica en este campo de la formación universitaria.

Problemáticas que van más allá de un análisis descontextualizado de las estrategias de enseñanza y de evaluación y que sólo encuentran su lugar propio en el campo disciplinar si se parte de un encuadre fundamentado crítico para el abordaje y la construcción de conocimiento acerca de las cuestiones de la enseñanza en la universidad.

\section{Notas}

1 Se entiende como prácticas pertinentes a aquellas que dan cuenta del principio de pertinencia social, esto es en su condición de estar en contacto con los ámbitos de la sociedad, tales como políticas estatales, mundo del trabajo, empresas, sistema educativo, tal como lo concibe UNESCO (1998).

2 Buchbinder (2005, p. 106) en su obra, Historia de las universidades argentinas, señala que en 1919 Alfredo Palacios (dirigente socialista y reformista) pronunció conferencias sobre el ideario reformista en Perú, desarrollándose un movimiento hacia el cambio de los estatutos universitarios, que se extendió a Chile, Paraguay y Cuba, culminando con la realización del Congreso Internacional de Estudiantes de 1921 en México, desde donde alcanzó proyección universal, especialmente en lo relativo al fortalecimiento de las organizaciones estudiantiles.

3 “[...] la transformación de la Universidad en un centro de formación científica a la par de ámbito de formación profesional constituyó un principio que aglutinó al grueso de la dirigencia reformista y que se vinculaba estrechamente con las orientaciones antiprofesionalistas" (BUCHBINDER, 2005, p. 123).

4 Estas instituciones de investigación, formación y servicios para la docencia universitaria, con numerosa producción escrita en la materia, tuvieron distintas denominaciones en esas más de tres décadas que transcurrieron desde su creación en la UNAM: primero fue el Centro de Investigaciones y Servicios Educativos (CISE), después el Centro de Estudios sobre la Universidad (CESU), y en la actualidad funciona como Instituto de Investigaciones sobre la Universidad y la Educación. (IISUE). 
5 María Isabel da Cunha (2009, p. 170) se refiere a esta problemática en sus escritos sobre la docencia y las innovaciones universitarias: “[...]a docência universitária recebeu forte influencia da concepção epistemológica dominante, própria da ciência moderna, especialmente inspiradora das chamadas ciências exatas e da natureza, que possuía a condição definidora do conhecimento socialmente legitimado. Nesse pressuposto, o conteúdo específico assumia um valor significativamente maior do que o conhecimento pedagógico e das humanidades na formação de professores".

6 Ovide Menin (1992), uno de los partícipes de aquellas unidades pedagógicas históricas mencionadas anteriormente, la de la Universidad Nacional del Litoral, en su obra Pedagogía y Universidad identifica problemáticas más cercanas al ámbito de de la Didáctica universitaria, aún cuando el autor no denomine así al campo: " La pedagogía universitaria es la teoría y la práctica de la enseñanza que se realiza de consuno en una institución concreta, llamada universidad. Sus requerimientos parten de un fenómeno social que implica la serie de aprendizajes singulares que realizan los adultos jóvenes" (MENIN, 1992, p. 15). Por su parte Denise Leite (2006, p. 57) parte también del reconocimiento de un único campo de estudios al señalar que "No contexto latino-americano, a Pedagogía Universitária, nucleada no campo educativo, opera no espaço transdiscilinar da academia. Tem como objeto de estudo o ensino, a aprendizagem e a evaluaçao na universidade".

7 En las reformas actuales de los planes de estudio en Argentina se advierte la persistencia de este pensamiento estrecho sobre el grupo de asignaturas más relacionadas con lo micro.

8 Cunha (2006, p. 22) señala al respecto "Outro aspecto da desqualificação da pedagogia universitária refere-se a sua composição instrumental e não raras vezes entendida como um conjunto de normas e prescriçóes que, na perspectiva da racionalidade técnica, teria um efeito messiânico na resolução de problemas".

9 Susana Garramuño (2000) en su Tesis además de la diversidad y la extranjeridad, incluye como categoría para comprender el ejercicio del rol del asesor pedagógico en la Universidad, el reconocimiento 
de la demanda como instituyente y por lo tanto como del pedido que pueda hacer el sujeto de asesoramiento, más ligado a lo instituido. La identificación de la demanda le permite al asesor pedagógico ir más allá de la queja paralizante que lo encerraría en el estancamiento de la dificultad sentida y en una posición asistencialista.

10 En el II Encuentro Nacional de Prácticas de Asesorías Pedagógicas Universitarias que organizamos en marzo del 2010, uno de los temas recurrentes en las exposiciones de los asesores pedagógicos universitarios, fue la tensión que se genera a partir de la coexistencia de distintos intereses y distintas perspectivas didácticas, muchas veces contradictorias, acerca de qué es enseñar y qué es aprender en la universidad, y por tanto hacia dónde debe orientar sus acciones el asesor.

11 El Instituto prácticamente no desarrolló actividades durante la dictadura de 1976-1983. A partir de 1984 es nombrada Directora María Teresa Sirvent, quien impulsa el retorno de los investigadores a este ámbito y abre las puertas del Instituto a las instituciones de la comunidad educativa, impulsando la realización de proyectos tendientes a la recuperación de dinámicas participativas en educación.

12 En junio de ese año asumo la coordinación de esas reuniones, dando así lugar al inicio de lo que al poco tiempo se organizó como Programa de Estudios sobre el aula universitaria.

13 Proyecto con sede en la Secretaría de Asuntos Académicos bajo la dirección de Lidia Fernández y con la participación, entre otros investigadores, de Claudia Finkelstein. Se realizaron estas Jornadas en 1991, 1992, 1993 y 1998, con la participación de alrededor de 400 docentes en cada una de ellas, y la presentación de 70 experiencias agrupadas en más de 20 de Talleres simultáneos. En ellos dos o tres equipos docentes de distintas facultades presentaban sus experiencias docentes alrededor de un mismo problema pedagógico.

14 En estas investigaciones participa el equipo integrado por Claudia Finkelstein, María E. Donato, Martha Nepomneschi, Gladys Calvo, Patricia Del Regno, Viviana Solberg, Mónica Gardel, Walter Viñas, con la dirección de Elisa Lucarelli. 


\section{REFERENCIAS}

BLEGER, J. Psicología de la conducta. Buenos Aires: Centro Editor de América Latina, 1971.

BUCHBINDER, P. Historia de las universidades argentinas. Buenos Aires: Sudamericana, 2005.

CANDAU, V. M. (Org.). Rumo a uma nova didáctica. Petrópolis: Vozes, 1995.

CUNHA, M. I. da (Org.). Pedagogia universitária: energias emancipatorias em tempos neoliberais. Araraquara: Junqueira \& Marin, 2006.

CUNHA, M. I. da. Inovaçôes pedagógicas na universidade. In: CUNHA, M. I. da; SOARES, S.; LOPES RIBEIRO, M. (Org.). Docência universitária: profissionalização e praticas educativas. Feira de Santana: Ed. da UEFS, 2009.

DÍAZ BARRIGA, A. Didáctica y currículum. Convergencia en los programas de estudio. México: Nuevomar, 1984.

DÍAZ BARRIGA, A. Ensayos sobre problemática curricular. México: Trilla-Anuies, 1990.

FERNÁNDEZ, L. Asesoramiento pedagógico institucional: una propuesta de encuadre de trabajo. Revista Argentina de Educación, Buenos Aires, año I, n. 2-3, 1982.

FERNÁNDEZ LAMARRA, N. La educación superior argentina en debate. Buenos Aires: Eudeba/IESALC, 2003.

FURLÁN, A. et al. Aportes a la didáctica de la educación superior. México: ENEPI/UNAM, 1979.

GARRAMUÑO, S. La construcción del rol del asesor pedagógico en el entorno académico de la FADU. 2000. Sin Publicar. Tesis (Maestría en Didácticas Específicas) - Facultad de Humanidades y Ciencias Universidad Nacional del Litoral, Santa Fe, 2000.

HELLER, A. Sociología de la vida cotidiana. Barcelona: Península, 1977.

LANGER, A. El sistema científico y las universidades: revisión de teorías en América Latina y Argentina. In: RIQUELME, G. (Ed.). Las 
universidades frente a las demandas sociales y productivas. Buenos Aires: Miño y Dávila, 2008. Tomo I.

LAFOURCADE, P. Planeamiento, conducción y evaluación en la enseñanza superior. Buenos Aires: Kapelusz, 1974.

LEITE, D. Pedagogia Universitária. In: ENCICLOPÉDIA DE PEDAGOGIA UNIVERSITÁRIA. Glossário. Brasilia: INEP/RIES, 2006. v. 2.

LUCARELLI, E. La didáctica de nivel superior. Buenos Aires: OPFyL, 1988.

LUCARELLI, E. Pedagogía Universitaria e Inovação. In: Cunha, M. I. da (Org.). Reflexóes e praticas em Pedagogia Universitária. Campinas: Papirus, 2007.

LUCARELLI, E. Análisis de prácticas y formación, tres experiencias con docentes en universidades argentinas. Perspectiva: Revista do Centro de Ciências da Educação, Florianópolis, v. 24, n. 1, jan./jun. 2006.

LUCARELLI, E. La teoría y la práctica en la universidad: la innovación en las aulas. Buenos Aires: Miño y Dávila, 2009.

MENIN, O. Pedagogía y universidad. Rosario: Homo Sapiens, 1992.

MOLLIS, M. Las huellas de la Reforma Educativa ante una nueva Ley de Educación Superior. Revista del IICE, Buenos Aires, año XVI, n. 26, p. 77-105, 2008.

NASSIF, R. Pedagogía universitaria y construcción de la universidad. Revista de la Universidad, La Plata, 1974.

NASSIF, R. Teoría de la educación. Madrid: Cincel-Kapelusz, 1980.

SIRVENT, M. T. Nuestra propuesta de trabajo: producto de una tarea compartida. Boletín del ICE, Buenos Aires, n. 3, 1985.

RENAUT, A. ¿Qué hacer con las universidades? Buenos Aires: Jorge Baudino; Ed. da UNSAM, 2008.

UNESCO. La educación superior en el siglo XXI: visión y acción. Paris, 1998. Documento de trabajo de la Conferencia Mundial sobre educación superior. 


\section{University pedagogy: a subject of interest for the university today?}

\section{Abstract}

The purpose of this article is to present the historic and current situation of the field of university pedagogy in Argentina, with special reference to the past 25 years and events in the institutional and national context. It exemplifies the problematics based on the characteristics and development of a research program about this issue, its lines of work and its production, emphasizing a study that looks at the current situation of education and the complex learning involved in the professional practice in the field of healthcare, particularly as undertaken in clinics. A qualitative methodology is used with case studies and instances of participation. The main results look at the characteristics that the teaching modalities assume, considering tension that is generated between the logic of education and the logic of healthcare, highlighting the identification of modalities developed by professors, in particular observation and supervision of the tasks of students, as they are engaged in real practices that anticipate professional performance. The modalities that are generated in relation to the place of students in education and the pedagogical derivations of the organization of the clinics and the cooperative learning among students are also considered.

\section{Didática universitária: um assunto de interesse para a universidade atual?}

\section{Resumo}

Este artigo tem como objetivo apresentar a situação histórica e atual do campo da Didática Universitária na Argentina, com especial referência aos últimos 25 anos e relacionados aos acontecimentos ocorridos no contexto institucional e nacional. Exemplifica suas problemáticas a partir das características e evolução de um programa de pesquisa naárea, suas linhas de trabalho e sua produção, enfatizando um estudo que analisa a atualidade sobre a formação em aprendizagens complexos da prática profissional na área da saúde, especialmente aqueles que se desenvolvem nas Clínicas. Com esse propósito, utiliza uma metodologia qualitativa com estudo de casos e níveis de participação. Seus principais resultados indicam as características que assumem as modalidades de ensino nessas áreas em termos de lidar com a tensáo gerada entre a lógica de formação e a lógica de atenção da saúde, destacando-se a identificação das modalidades desenvolvidas pelo professor, em especial a observação e a supervisão da tarefa dos estudantes, como prática real antecipatória do desempenho profissional. Da mesma maneira, foram reconhecidos padróes gerados em relação ao lugar do estudante na situação de formação e as derivaçóes didáticas da organização nas 
Keywords: Pedagogy - Higher clínicas e na aprendizagem cooperativa education. Educational intervention. entre estudantes.

Innovation in Education. Palavras-chave: Didática - Educação Superior. Intervenção Educativa. Inovação em Educação.

\section{Elisa Lucarelli}

Instituto de Investigaciones en Ciencias de la Educación. FFyL.UBA. Puan 4804to. Piso Ciudad de Buenos Aires, Argentina.

Telefones: (005411) 4432-0606 (ext. 119) / 4432-0212 (Fax)

E-mail: elisalucarelli@arnet.com.ar

Recebido em: $16 / 9 / 2010$

Versão final recebida em: 22/10/2011

Aprovado em: 30/10/2011 